\title{
THE ABSOLUTE CONTINUITY OF A CLASS OF TOEPLITZ OPERATORS
}

\author{
MARVIN ROSENBLUM ${ }^{1}$
}

\begin{abstract}
It is shown that certain generalized Toeplitz operators are the real parts of subnormal operators that have no normal part, and hence are absolutely continuous. It follows that the Toeplitz operators associated with the real parts of nonconstant bounded holomorphic functions in the polydisc are absolutely continuous.
\end{abstract}

1. Introduction. Let $\sigma$ be normalized Lebesgue measure on the unit circle $\Gamma$, so $L^{2}(d \sigma)$ has $\left\{\chi^{n}\right\}_{n=-\infty}^{\infty}$ as a complete orthonormal basis, where $\chi\left(e^{i \phi}\right)=e^{i \phi}$. Let $\boldsymbol{P}$ be the orthogonal projection on the closed linear span $H^{2}$ of $\left\{\chi^{n}\right\}_{n=0}^{\infty}$ and fix a real function $w$ in $L^{\infty}(d \sigma)$. Then the operator $\boldsymbol{T}$ on $H^{2}$ defined by $\boldsymbol{T} f=\boldsymbol{P}(w f), f \in H^{2}$, is the selfadjoint Toeplitz operator associated with $w$. Now, a selfadjoint operator is said to be AC (absolutely continuous) if its spectral measure is weakly absolutely continuous (with respect to linear Lebesgue measure). It was proved in [5] that if $\boldsymbol{T}$ is not a multiple of the identity, then $\boldsymbol{T}$ is AC. The proof in [5] depends on an explicit representation for the resolvent of $\boldsymbol{T}$, and does not appear to admit a ready generalization. However there is a remarkable "soft analysis" proof by C. R. Putnam [4, pp. 131-133] that $T$ is AC under the added assumption that $w$ is the real part of a nonconstant $H^{\infty}$ function. We shall employ Putnam's method and set down a large class of AC generalized Toeplitz operators by generalizing Theorem 6.6.1 of [4], which is due to R. G. Douglas.

Suppose $(X, B, \mu)$ is a measure space and $\mathscr{H}$ is a (closed) subspace of $L^{2}(d \mu)$. Let $\boldsymbol{P}$ be the projection of $L^{2}(d \mu)$ on $\mathscr{H}$. Assume that every function $f$ in $\mathscr{H}$ that vanishes on a set of positive measure vanishes a.e. Then an operator $\boldsymbol{T}$ on $\mathscr{H}$ of the form $\boldsymbol{T} f=\boldsymbol{P}(w \cdot f), f \in \mathscr{H}$, where $w$ is a fixed real function in $L^{\infty}(d \mu)$, is a generalized Toeplitz operator. For a different generalization of a Toeplitz operator see [2].

Received by the editors August 21, 1972.

AMS (MOS) subject classifications (1970). Primary 47B35; Secondary 47B20.

Key words and phrases. Toeplitz operator, subnormal operator, absolutely continuous operator.

${ }^{1}$ Research supported by NSF Grant GP-31483X.

c) American Mathematical Society 1973 
2. Real part of a hyponormal operator. Throughout $\mathscr{H}$ is a complex Hilbert space and $\mathscr{B}(\mathscr{H})$ is the space of bounded operators on $\mathscr{H}$ to $\mathscr{H}$. An operator $\boldsymbol{A}$ in $\mathscr{B}(\mathscr{H})$ is hyponormal if $\boldsymbol{A}^{*} \boldsymbol{A} \geqq \boldsymbol{A} \boldsymbol{A}^{*}$. $\boldsymbol{A}$ has no normal part if there is no nonnull subspace $\mathscr{M} \subseteq \mathscr{H}$ such that $\mathscr{M}$ reduces $A$ and $\boldsymbol{A} \mid \mathscr{M}$ is a normal operator.

Theorem 1 (C. R. Putnam). If $\boldsymbol{A}$ is a hyponormal operator with no normal part, then $A+A^{*}$ is $A C$.

Proof. [4, pp. 131-133].

3. The F. and M. Riesz property. Let $A$ be a subnormal operator in $\mathscr{B}(\mathscr{H})$, so there exists a Hilbert space $\mathscr{K}$ containing $\mathscr{H}$ as a subspace and a normal operator $N$ in $\mathscr{B}(\mathscr{K})$ such that $\mathscr{H}$ is an invariant subspace of $\boldsymbol{N}$ and $\boldsymbol{A}=\boldsymbol{N} \mid \mathscr{H}$. Let $\boldsymbol{P}$ be the projection of $\mathscr{K}$ on $\mathscr{H}$. Necessarily $\boldsymbol{A}$ is hyponormal. In order to apply Theorem 1 we set down a sufficient condition for $\boldsymbol{A}$ to have no normal part.

Definition 1. Suppose $N$ is a normal operator in $\mathscr{B}(\mathscr{K})$ with spectral representation. $\boldsymbol{N}=\int z \boldsymbol{E}(d z)$, where $\boldsymbol{E}$, the spectral measure of $\boldsymbol{N}$, is defined on all planar Borel sets. $(N, \mathscr{H})$ has the $F$. and $M$. Riesz property if whenever $\boldsymbol{E}(\Delta) f \in \mathscr{H}$ for all Borel sets $\Delta$, then $f=0$.

THEOREM 2. Suppose $A$ is a subnormal operator in $\mathscr{B}(\mathscr{H})$ and $(N, \mathscr{H})$ has the $F$. and $M$. Riesz property. Then $\boldsymbol{A}$ has no normal part and $\boldsymbol{A}+\boldsymbol{A}^{*}$ is $A C$.

Proof. Suppose $(N, \mathscr{H})$ has the F. and M. Riesz property and that the subnormal operator $A$ is normal on a subspace $\mathscr{M}$ of $\mathscr{H}$. If $f \in \mathscr{M}$, $\boldsymbol{N} f=\boldsymbol{A} f \in \mathscr{M}$ and $\left\|\boldsymbol{P} \boldsymbol{N}^{*} f\right\|=\left\|\boldsymbol{A}^{*} f\right\|=\|\boldsymbol{A} f\|=\|\boldsymbol{N} f\|=\left\|\boldsymbol{N}^{*} f\right\|$, so $\boldsymbol{N}^{*} f \in \mathscr{M}$. Thus $\mathscr{M}$ reduces $N$, and it follows that $F(\Delta) \mathscr{M} \subseteq \mathscr{M}$ for all planar Borel sets $\Delta$. Thus by the F. and M. Riesz property $\mathscr{M}=\{0\}$, so $A$ has no normal part. By Theorem $1, \boldsymbol{A}+\boldsymbol{A}^{*}$ is AC.

4. A class of AC generalized Toeplitz operators. We shall apply Theorem 2 to a concrete situation. $(X, B, \mu)$ is a measure space and $\mathscr{H}$ is a subspace of $L^{2}(d \mu)$.

THEOREM 3. Let $n \in L^{\infty}(d \mu)$ and assume $n$ is not a.e. constant. Let $N$ be the operator of multiplication by $n$ on $L^{2}(d \mu)$. Assume that $\mathscr{H}$ is an invariant subspace of $N$ with the property that whenever an $f$ in $\mathscr{H}$ vanishes on a set of positive $\mu$-measure, then $f=0 \mu$-a.e.

Then $\boldsymbol{A}=\boldsymbol{N} \mid \mathscr{H}$ has no normal part and $\boldsymbol{A}+\boldsymbol{A}^{*}$ is an $A C$ generalized Toeplitz operator.

Proof. $\quad N$ is not a multiple of the identity, so there exists a Borel set $\Delta$ such $I \neq E(\Delta) \neq 0$. Since $N$ is a multiplication operator, so is $\boldsymbol{E}(\Delta)$, and 
$E(\Delta)$ is necessarily multiplication by the characteristic function $\chi_{\beta}$ of a measurable set $\beta$ with $\mu(\beta) \neq 0, \mu(X-\beta) \neq 0$. We shall show that $(N, \mathscr{H})$ has the F. and M. Riesz property. Suppose $f \in \mathscr{H}$ and $\boldsymbol{E}(\Delta) f \in \mathscr{H}$. Then $\chi_{\beta} \cdot f$ vanishes off $\beta$, so $\chi_{\beta} \cdot f=0$ a.e. But then $f=0$ a.e. Theorem 3 now follows from Theorem 2 .

5. Examples. We note that a generalized Toeplitz operator is of the form $\boldsymbol{T} f=\boldsymbol{P}(w \cdot f), f \in \mathscr{H}, w \in L^{\infty}(d \mu)$, whereas Theorem 3 only speaks to the situation when $w=n+\bar{n}$, where $n \mathscr{H} \subseteq \mathscr{H}, n \in L^{\infty}(d \mu)$. In Example 1 below every generalized Toeplitz operator that is not a multiple of the identity is AC, but this is not the case in Examples 2 and 3.

EXAmple 1. Let $\sigma, \mathscr{H}=H^{2}, \boldsymbol{T}$ be as in the first part of the introduction and see [5].

Example 2 (M. B. Abrahamse [1]). Fix $\rho \in(0,1)$ and let $\Gamma_{\rho}=$ $\{z: z$ complex, $|z|=\rho\}, \Gamma=\{z: z$ complex, $|z|=1\}$. Let $\mu$ be the measure on $\Gamma \cup \Gamma_{\rho}$ such that $\mu \mid \Gamma$ and $\mu \mid \Gamma_{\rho}$ are normalized Lebesgue measures on $\Gamma$ and $\Gamma_{\rho}$ respectively. $\mathscr{H}=H^{2}$ is defined to be the closed linear span of $\left\{z^{n}\right\}_{n=-\infty}^{\infty}$ in $L^{2}(d \mu)$ and $H^{\infty}$ is defined to be the weak star closure of $\left\{z^{n}\right\}_{n=-\infty}^{\infty}$ in $L^{\infty}(d \mu)$.

By Theorem 3, generalized Toeplitz operators $\boldsymbol{T}$ of the form $\boldsymbol{T} f=$ $\boldsymbol{P}(w \cdot f), f \in H^{2}$, where $w=\operatorname{Re} n, n \in H^{\infty}, n$ not a.e. constant, are AC. However, as shown by Abrahamse, in case $w(z)=1$ if $z \in \Gamma$ and $w(z)=0$ if $z \in \Gamma_{\rho}$, the associated generalized Toeplitz operator has pure point spectra. This counterexample was noted by Muhly [3] in his generalization of Putnam's method.

EXAmple 3. Consider the $n$-torus

$$
\left.\Gamma^{(n)}=\left\{e^{i \phi_{1}}, e^{i \phi_{2}}, \cdots, e^{i \phi_{n}}\right): e^{\phi_{1}}, \cdots, e^{i \phi_{n}} \in \Gamma\right\}
$$

with Lebesgue measure $d \mu=(2 \pi)^{-n} d \phi_{1} \cdots d \phi_{n}$. Let $\mathscr{H}=H^{2}$ be the closed linear span of $\mathscr{A}=\left\{e^{i j_{1} \phi_{1}} \cdots e^{i j_{n} \phi_{n}}\right\}_{j_{1}, \cdots, j_{n}}^{\infty}=0$ in $L^{2}(d \mu)$ and $H^{\infty}$ the weak star closure of $\mathscr{A}$ in $L^{\infty}(d \mu)$.

Now [6, p. 325], an $f$ in $H^{2}$ that vanishes on a set of positive measure vanishes a.e. so generalized Toeplitz operators associated with the real parts of nonconstant $H^{\varkappa}$ functions are AC. However not every generalized Toeplitz operator is AC. For example, let $n=2$ and $w\left(e^{i} \phi, e^{i} \psi\right)=$ $\cos (\phi-\psi)$. Then $\boldsymbol{T} 1=0$, so $\boldsymbol{T}$ has point spectrum.

We conclude with a theorem of Virginia Rovnyak, which we include with her permission.

THEOREM 4. Let $\mathscr{H}=H^{2}$ of the unit circle $\Gamma$, suppose $h$ is $H^{\infty}$ and is not a.e. constant, and let $\boldsymbol{S}$ be the shift operator, $S f=\chi f, f \in H^{2}$. Then the closed linear span of $\left\{\boldsymbol{S}^{* j} h^{k}: j, k=1,2,3, \cdots\right\}$ is $\mathscr{H}$. 
Proof. Define $\boldsymbol{A}$ on $H^{2}$ and $\boldsymbol{N}$ on $L^{2}(d \sigma)$ as multiplication by $h$. To prove the result it suffices to show that $\mathscr{L}=H^{2} \ominus\left\{S^{* j} h^{k}: j, k=1,2,3, \cdots\right\}$ reduces $N$. In that case $\mathscr{L}$ reduces $A$ and $A|\mathscr{L}=N| \mathscr{L}$ is normal, so $\mathscr{L}=0$ by Theorem 2 .

Clearly $S \mathscr{L} \subseteq \mathscr{L}$, so $\quad \boldsymbol{N} \mathscr{L} \subseteq \mathscr{L}$. If $f \in \mathscr{L}, n \geqq 1,\left\langle\boldsymbol{N}^{*} f, \chi^{-n}\right\rangle_{L^{2}}=$

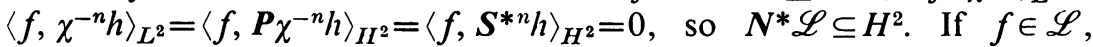
$j, k \geqq 1,\left\langle N^{*} f, S^{* j} h^{k}\right\rangle_{H^{2}}=\left\langle N^{*} f, \chi^{-j} h^{k}\right\rangle_{L^{2}}=\left\langle f, \chi^{-j} h^{k+1}\right\rangle_{L^{2}}=\left\langle f, S^{* j} h^{k+1}\right\rangle_{H^{2}}=$ 0 , so $N^{*} \mathscr{L} \subseteq \mathscr{L}$. We have shown that $\mathscr{L}$ reduces $N$, as required.

\section{REFERENCES}

1. M. B. Abrahamse, Toeplitz operators in multiply connected regions, Bull. Amer. Math. Soc. 77 (1971), 449-453. MR 42 \#8313.

2. R. G. Douglas and C. Pearcy, Spectral theory of generalized Toeplitz operators, Trans. Amer. Math. Soc. 115 (1965), 433-444. MR 33 \#7849.

3. P. S. Muhly, Maximal weak-* Dirichlet algebras and selfadjoint Toeplitz operators (preprint).

4. C. R. Putnam, Commutation properties of Hilbert space operators and related topics, Ergebnisse der Mathematik und ihrer Grenzgebiete, Band 36, Springer-Verlag, New York, 1967. MR 36 \#707.

5. M. Rosenblum, The absolute continuity of Toeplitz's matrices, Pacific J. Math. 10 (1960), 987-996. MR 22 \#4952.

6. A. Zygmund, Trigonometrical series. Vol. 2, 2nd ed., Cambridge Univ. Press, New York, 1959. MR 21 \#6498.

Department of Mathematics, University of Virginia, Charlottesville, Virginia 22903 